\title{
A NOVEL APPROACH TO PERINATAL HIV/AIDS TRAINING FOR DOCTORS AND NURSES IN SOUTH AFRICA
}

\author{
David L Woods, $M D$, FRCP, $D C H$ \\ Division of Neonatal Medicine, School of Child and Adolescent Health, University of Cape Tocon
}

\begin{abstract}
The large number of children presenting with AIDS in South Africa is a wake-up call for urgent attention to be paid to reducing the risk of mother-to-child transmission of HIV. This requires an innovative method of training the thousands of nurses and doctors who provide perinatal care, especially in rural areas with limited opportunities for ongoing education. The Perinatal Education Programme is a self-help method of learning which has been very successful in enabling nurses and doctors to take responsibility for their own distance training. A recent Perinatal HIV/AIDS supplement to the Programme provides a novel and appropriate means of meeting this training need.
\end{abstract}

As the HIV epidemic sweeps across South Africa, many of the AIDS victims are young children infected by their mothers in the perinatal period. While these children unfortunately bear the brunt of the early epidemic, many of them could have been spared the horrors of AIDS if more attention had been paid to reducing the risk of vertical transmission. Screening during pregnancy, short-course antiretroviral prophylaxis, modification of the management of labour and delivery, and exclusive breast- or formulafeeding promise to significantly reduce the rate of motherto-child transmission of HIV and therefore the incidence of childhood AIDS. However, to introduce these essential changes in management, large numbers of nurses and doctors will require additional training. This is problematic due to the lack of funding, few available tutors and the impracticality of moving staff to regional centres for training courses. Traditional methods of centralised teaching with tutors training small groups of students in teaching hospitals are expensive and no longer appropriate or applicable. What is required is an innovative method of enabling nurses and doctors to manage their own continuing education. This need is particularly urgent with staff caring for pregnant women who are HIV-infected and their HIV-exposed infants.

The Perinatal Education Programme (PEP) offers a cheap and practical means of addressing the problem.' PEP is a self-help distance learning course that aims at improving the care of pregnant women and their newborn infants, especially in rural areas. It is presented in the form of training manuals that use a problem-orientated, patientbased approach. A question-and-answer method of problem solving is used as the basic learning model: first a question is asked and then the appropriate answer is given. Utilising this method, participants are led from the most basic facts to more complex concepts. Case studies are also used to place the information into clinical context. Participants find this method of outreach education simple, easy and enjoyable. Flow diagrams are used to summarise diagnostic and management protocols while a multiple choice test, before and after each unit, allows the participants to monitor their own progress. Skills workshops include simple line drawings and step-by-step instructions to master clinical skills. Widespread consultation with colleagues and comment by course participants has transformed PEP into a consensus statement of maternal and newborn care that has helped to standardise the provision of perinatal care in South Africa.

The standard PEP course consists of two training manuals, one on maternal and the other on newborn care. Each manual has 15 units, addressing important or common clinical problems. A manual usually requires a year to complete. Groups of 5 - 10 students study the Programme together. They elect a group leader who manages the course and arranges a meeting of participants every 3 weeks. A formal tutor is not required. The course material from one unit at a time is studied by each participant at home and then discussed at the group meetings, thus introducing the concept of peer tuition. Group members encourage, support and teach each other in a spirit of cooperative learning. Participants complete and mark their own multiple choice tests, enabling them to monitor their own progress through the course. The responsibility of learning is therefore placed squarely on the shoulders of 
each participant. The group leader invites an appropriate medical or nursing colleague to attend the PEP meeting in order to demonstrate the clinical skills attached to the unit being studied. In this way local resource personnel are encouraged to share their clinical abilities, and solve the seemingly insurmountable problem of skills instruction in rural areas.

A formal multiple-choice examination is offered to participants on completion of each manual. Regional health care services manage their own examination. Certificates are awarded to successful candidates and a graduation ceremony is arranged that acknowledges the considerable effort of the participants, and encourages other colleagues to join a PEP group. Participants buy their own manuals from the Perinatal Education Trust at R150 each. This payment allows the Trust, which produces the Programme, to be financially self-sufficient. Through the generosity of a private donor, nurses from the public sector who pass the PEP examination may apply for a retrospective bursary that repays the cost of their course. This system rewards success and enables most practising midwives in South Africa to join the project. Because each group takes ownership of their course, the pride and sense of achievement is theirs.

To date more than 30000 PEP manuals have been distributed and over 10000 certificates awarded to nurses and medical practitioners, as well as undergraduate nursing and medical students, who have successfully completed the course in southern Africa. A field study and two prospective controlled trials of the Maternal Care and Newborn Care PEP manuals have documented a significant improvement in cognitive knowledge, clinical skills, attitudes and patient care practices of midwives in South Africa. ${ }^{2-7}$ In the prospective trials, either the maternal or newborn care manual was introduced into a district hospital and its primary care clinic while the PEP was withheld from the neighbouring district, which was used as a control. Midwives in both the intervention and control districts were objectively assessed at the start and again at the end of the study. The midwives using PEP managed their own course over 12 months without a trainer. This is the first study to have documented that groups of midwives can be empowered to enhance their own clinical knowledge and skills and improve their attitudes and standard of patient care without the infrastructure of a formal training institution. Midwives using PEP reported that they felt more confident in their clinical abilities, experienced less stress at work, and achieved a greater degree of job satisfaction. Perhaps the awareness and appreciation of self-growth is one of the most important achievements of this method of distance education.

Recently a supplementary PEP manual on Perinatal
HIV/AIDS has been developed. It allows groups of professional health care workers to learn about all aspects of perinatal HIV infection. The five units address the following topics:

A general introduction to HIV infection and AIDS.

- Management of HIV-infected women during pregnancy.

- Management of HIV-infected women during labour and delivery.

- Management of HIV-exposed infants.

- Counselling HIV-positive women.

The learning material provides the reader with the basic theoretical knowledge of HIV infection and AIDS, an approach to diagnosis and management of mother and infant, and counselling principles to support the mother and family. Thirteen experts in the field of perinatal HIV in South Africa contributed to the development of the training manual. The layout and method of learning is the same as the two basic PEP manuals. The cost of the Perinatal HIV/AIDS manual is R50, inclusive of VAT, postage and packaging, and the end-of-course examination. A certificate is awarded to candidates who successfully complete the 4-month course and pass the final multiplechoice examination. The course offered by the Perinatal HIV/AIDS manual is recognised for $10 \mathrm{CPD}$ points provided the doctor successfully completes the examination. Both the standard manuals and the supplementary manual on Perinatal HIV/AIDS are available in either English or Afrikaans.

In a small field trial in KwaZulu/Natal, midwives studied the Perinatal HIVIAIDS manual. An assessment of their knowledge of the subject, before and after they conducted their own self-help course, showed a significant improvement from $64 \%$ to $94 \%$. This result is similar to the more exhaustive assessments of the two basic PEP manuals. Over the past year, 1000 Perinatal HIV/AIDS manuals have been distributed and well received by doctors and nurses working in both hospitals and clinics.

The Perinatal Education Programme offers a cheap and appropriate means of bringing on-site training to the many thousands of professional health care workers who are struggling to provide adequate care to HIV-positive women and their newborn children, especially in rural areas. The cost of the course is minimal compared with that of traditional training methods, and no formal teaching infrastructure is needed. Provisional results suggest that the Perinatal HIV/AIDS manual is as effective in providing appropriate knowledge as the rest of the Perinatal Education Programme. What is required is a concerted national and provisional plan to promote this form of educational opportunity to all who could benefit. With well-trained midwives and doctors, and a simple, appropriate protocol of managing pregnant women who 
are HIV-positive, the present high rate of mother-to-child transmission can be reduced, with a resultant drop in the numbers of children suffering from and dying of AIDS in South Africa.

For further information visit the Perinatal Education Trust website at www/pepcourse.co.za or contact the Trust by phone or fax at (021) 671-8030.

\section{REFERENCES}

Woods DL. An innovative programme for training in maternal and newborn care. Semin Neonatol 1999; 4: 209-216.
2. Woods DL, Theron GB. The impact of the Perinatal Education Programme on cognitive knowledge in midwives. S Afr Med J 1995; 85: 150-153.

3. Theron GB. Improved cognitive knowledge of midwives practicing in the Eastern Cape Province of the Republic of South Africa through study of a self educationa manual. Midwifery 1999; 15: 66-71.

4. Theron $\mathrm{GB}$. Improved practical skills of midwives practicing in the Eastern Province of the Republic of South Africa through the study of a self-education manual. J Perinatol 2000; 3: 184-188.

5. Theron GB. The effect of the Maternal Care manual of the Perinatal Education Programme on the attitude of midwives towards their work Curotionis 1999 December: 63-67.

6. Theron GB. The effect of the Maternal Care Manual from the Perinatal Education Programme on the quality of ante- and intrapartum care rendered by midwives. S Afr Med I 1999; 89: 336-342.

7. Greenfield DH. Evaluation of the use of the Neonatal Manual of the Perinata Education Programme. In: Proceedings of the 7th World IAMANEH Conference Pretoria: University of Pretoria, 1999: 229-231.

\section{INDUSTRY NEWS}

\section{Introduction of the Bristol-Myers Squibb Anti-Infective Franchise}

The Castle in Kyalami was the ideal venue for Bristol-Myers Squibb to introduce the newly formed Anti-Infective Franchise in both grandeur and style on 3 December 2002. The sovereign feast and dancing were enjoyed by all until well into the night.

Bristol-Myers Squibb have a wealth of knowledge in the anti-infective market with products including Tequin, Maxipime, Prozef, Fungizone, Cefril, Cefril-A, Cefacidal and Azactam in their Anti-Infective Franchise.

In addition, Bristol-Myers Squibb has a strong AntiRetroviral Franchise in Zerit and Videx, which are supplied below cost to the South African market.

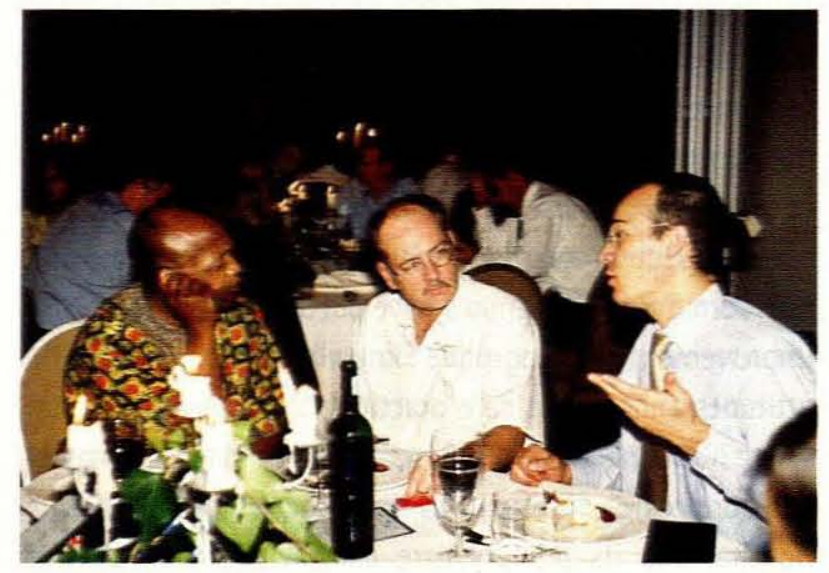

Dr $O$ Mwanthembe, Prof. G Richards, Dr M Sussman.

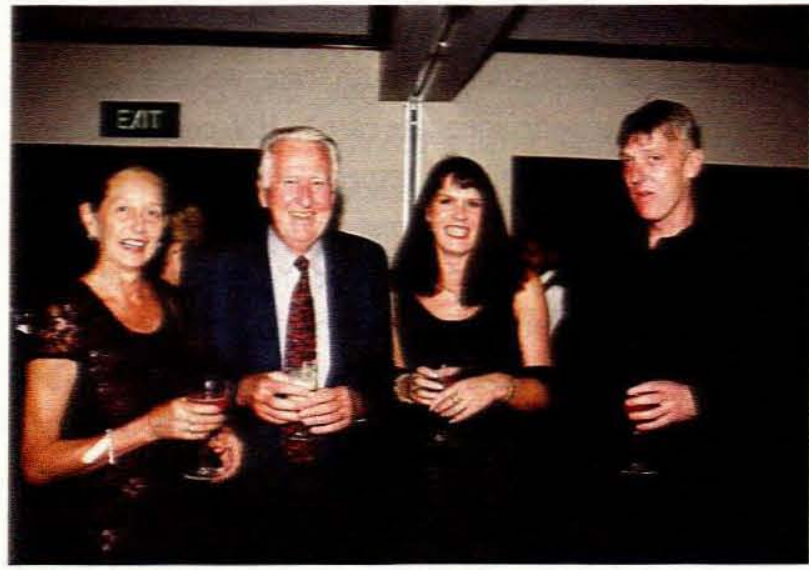

Mrs and Prof. De K Sommers, Ms R Coetzee (Prozef Product Manager), DrA Brink.

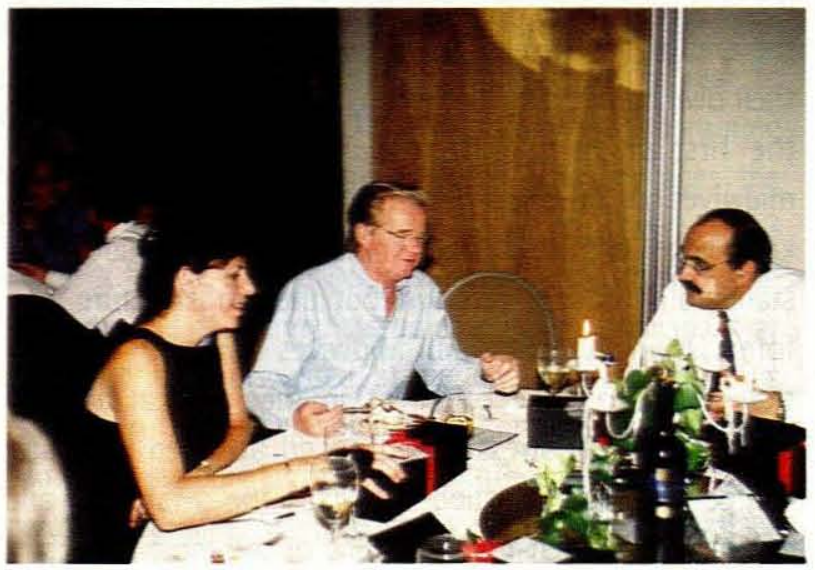

Ms L Mackenzie (BMS Anti-Infective Franchise Manager), Mr I Strachan (CEO of BMS), Prof. CFeldman. 